\title{
Hierarchical Spidroin Micellar Nanoparticles as the Precursors of Spider Silks
}

Lucas R. Parent ${ }^{1,2^{*}}$, David Onofrei ${ }^{3}$, Dian $\mathrm{Xu}^{4}$, Dillan Stengel ${ }^{3}$, John D. Roehling ${ }^{5}$, J. Bennett Addison ${ }^{3}$, Christopher Forman ${ }^{2}$, Samrat A. Amin ${ }^{4}$, Brian R. Cherry ${ }^{4}$, Jeffery L. Yarger ${ }^{4}$, Nathan C. Gianneschi ${ }^{2}$ and Gregory P. Holland ${ }^{3}$

1. University of Connecticut, Innovation Partnership Building, Storrs, CT, USA.

2. Northwestern University, Depts. of Chemistry, Materials Science, and Biomedical Engineering, Northwestern University, Evanston, IL, USA.

3. San Diego State University, Dept. of Chemistry and Biochemistry, San Diego, CA, USA.

4. Arizona State University, School of Molecular Sciences and the Magnetic Resonance Research

Center, Tempe, AZ, USA.

5. Lawrence Livermore National Laboratory, Materials Science Division, Livermore, CA, USA.

* Corresponding author: Lucas.Parent@uconn.edu

The process by which spiders store large silk proteins (spidroins) in high concentration in their glands and then spin them through their ducts into some of the strongest and toughest tensile materials known remain a critical question for the development of high-performance bioinspired fibrous materials [1]. Through decades of research, the primary sequences of the predominant natural silk spidroins have been determined, several biochemical triggers (e.g. $\mathrm{pH}$ change and shear) of the spinning process have been identified and can be artificially mimicked, and the microstructure of naturally-spun and artificial silk fibers have been well characterized [1]. However, the mechanism(s) that enable high concentration protein storage in the duct, and the subsequent processes of transformation into solid [polycrystalline] fiber that occurs in the spinning duct remain largely mysterious. It is theorized, for example, that the spidroins, being generally amphiphilic, pack into micelle-like species ("micelle theory") during storage in the spider's gland [2, 3], and are then funneled through the spider's spinning apparatus where they are transformed into liquid crystalline microstructures and ultimately into solid silk fibers. Despite the support in the field for the micelle theory of spidroin storage, we have lacked direct evidence that validate this theory and verify the existence of micellar nanoparticles in the silk dope precursor of spiders.

In this work, we have used a combination of two complementary nanoscale characterization techniques to test the micelle theory $[2,3]$ and investigate the initial steps of transformation that occur when the silk protein dope solution is extruded/sheared. We analyzed the natural protein dope from the silk glands of Black Widow spiders to determine the physical form of the liquid precursor of natural silk fibers. We use a combination of direct and indirect characterization methods, applying solution NMR spectroscopy and cryo-TEM tomographic imaging using discrete 3D reconstruction and visualization (discrete algebraic reconstruction technique, DART). Measuring the diffusion behavior of the spidroins in the natural silk dope by NMR reveals that the spidroins in the silk precursor are predominantly confined within entangled volumes of several-hundred $\mathrm{nm}$ diameters, suggesting the existence of tightly-packed spherical micelles, as postulated in the existing micelle theory $[2,3]$. High-resolution 3D imaging of these nanostructures by cryo-TEM tomography (Figure 1) shows that the spidroin micellar assemblies are far more morphologically complex than previously postulated, existing as hierarchical micellar nanoparticles ( 100-400 nm diameters) composed of networks of flake-like subdomains [4]. When the native dope solution is physically sheared, mimicking the extrusion process through a spider's spinning duct, we find that the subdomains within the hierarchical micelles transform, become narrowed and elongated fibrils that remain aggregated as hierarchical interwoven assemblies [4]. 
The cryo-TEM results show evidence that the existing "micelle theory" of silk fiber seeding is only partially correct, and has missed the subtle nanoscale structural details that could be critical for the initial protein precursor-to-fiber transformation. We propose a modified micelle theory, whereby the glandstored precursors exist as complex, hierarchical assemblies $(\sim 500 \mathrm{~nm}$ compound micelles with $\sim 50 \mathrm{~nm}$ subdomains), not as simple spherical micelles. With this fundamental scientific understanding of the natural system, we hypothesize that synthetic silks are also best spun from hierarchical micellar nanoparticles (200-500 $\mathrm{nm}$ diameters) of silk proteins rather than from a disperse or randomly aggregated soup, or from "simple" (non-hierarchical) spherical particles [5].

\section{References:}

[1] JL Yarger, BR Cherry and A van der Vaart, Nat. Rev. Mater. 3 (2018), p. 8000.

[2] F Hagn et al., Nature 465 (2010), p. 239.

[3] H-J Jin and DL Kaplan, Nature 424 (2003), p.1057.

[4] LR Parent et al., Proc. Natl. Acad. Sci. 115 (2018), p. 11507.

[5] LRP was supported by the National Institute of Biomedical Imaging and Bioengineering of the National Institutes of Health under Award F32EB021859. J.D.R.'s work was performed under the auspices of the US Department of Energy, by Lawrence Liver- more National Laboratory under Contract DE-AC52-07NA27344. Grants that supported this work include: (DOD-AFOSR) FA9550-171-0282 (to G.P.H.), (DURIP) FA9550-17-1-0409 (to G.P.H.), (NSF-DMR-BMAT) 1809645 (to J.L.Y.), and (ARO MURI) W911NF-15-1-0568 (to N.C.G.).
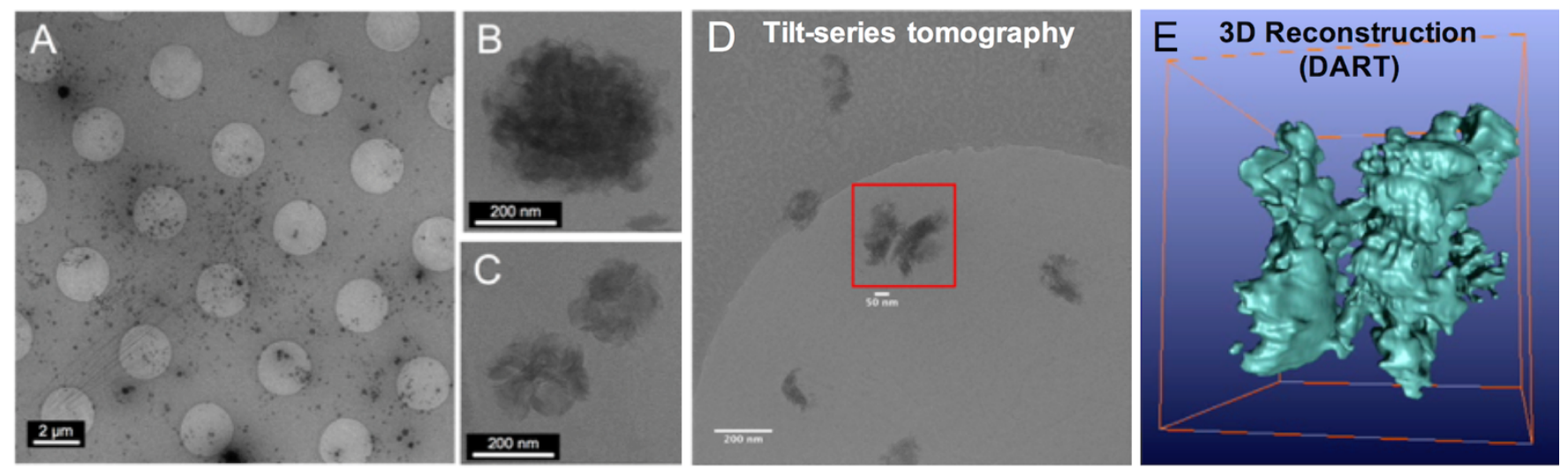

Figure 1. Native silk protein "dope" of the L. hesperus (black widow) spider imaged by low dose cryoTEM. (A) Low-mag image: the silk proteins in the highly concentrated dope solution take the form of generally spherical $(200-400 \mathrm{~nm})$ micellar assemblies. (B,C) High-mag images of the silk protein assemblies, which are hierarchical structures composed of disordered "flake-like" sub-domains. (D) CryoTEM tomography tilt-series of a selected hierarchical micellar assembly. (E) 3D isosurface rendering (using discrete algebraic reconstruction technique) of the selected assembly in D. 